\title{
Epidemiology of fatal cases associated with pandemic influenza reported in Yemen
}

\author{
Ahmed A. K. Thabet ${ }^{1 \#}$, Najeeb M. Moulhee ${ }^{2}$, Abdulhakeem Al-kohlani $^{3}$, Mohammed Jahaf ${ }^{3}$ \\ ${ }^{1}$ Community Medicine Department, Faculty of Medicine \& Health Sciences, Thamar University, Dhamar, Yemen; \\ \#Corresponding Author: thabet64@live.com \\ ${ }^{2}$ Faculty of Medicine and Health Sciences, Hodiedah University, Hodiedah, Yemen \\ ${ }^{3}$ Diseases Control and Surveillance, Ministry of Public Health \& Population, Sana'a, Yemen
}

Received 9 September 2012; revised 12 October 2012; accepted 24 October 2012

\section{ABSTRACT}

Objectives: This study describes the incidence of all fatal cases associated with Pandemic Influenza A (H1N1) in Yemen. It sets out to highlight the factors associated with poor prognosis to enhance the implementation of prevention and control programs. Methods: The study is based on retrospective analysis of available data until 14 March 2010, as compiled by the disease control and surveillance team in Yemen. Results: Between 16 June 2009 and 14 March 2010, a total of 33 laboratory-confirmed death cases associated with pandemic influenza A (H1N1) were reported to the Diseases Control and Surveillance in the Ministry of Public Health and Population. During this period, a total of 6049 suspected influenza $A$ (H1N1) cases were recorded. With this denominator, the case fatality rate (CFR) was $0.54 \%$. During June through August, H1N1 confirmed cases were infrequently detected, including only 30; however, from September through December, over 200 confirmed cases were reported each month. Of the 33 cases recorded, 25 were male $(76 \%)$ and 8 were female $(24 \%)$, male to female ratio being $3: 1$. Overall median age of the death cases was $\mathbf{3 0 . 8}$ years (range 1 - 55). The most common diagnosis upon admission was pneumonia. Out of the deaths, twenty five $\mathbf{( 7 5 . 8 \% )}$ had no documented underlying diseases. Chronic cardiovascular disease (9.1\%) was the most commonly reported disease and 2 deaths (6.1\%) were recorded as pregnant women. Conclusions: The most common diagnosis upon admission was pneumonia. Chronic cardiovascular diseases were the most commonly reported underlying conditions, while the most identified risk factor was pregnancy. These findings should be taken into considera-

\footnotetext{
*The authors declare that they have no competing interests.
}

tion, when vaccination strategies are employed.

Keywords: Pandemic Influenza (H1N1) 2009; Fatal Cases; Yemen

\section{INTRODUCTION}

Influenza A and B are the two types of influenza viruses that cause epidemic human disease. Influenza $\mathrm{A}$ viruses are categorized into subtypes on the basis of two surface antigens: hemagglutinin and neuraminidase. Influenza A subtypes and B viruses are separated further into groups on the basis of antigenic similarities. New influenza virus variants result from frequent antigenic change (i.e., antigenic drift) caused by point mutations and recombination events that occur during viral replication. Recent studies have explored the complex molecular evolution and epidemiologic dynamics of influenza A viruses. New or substantially different influenza A subtypes have the potential to cause a pandemic when they are able to cause human illness and demonstrate efficient human-to-human transmission and when little or no previously existing immunity has been identified among humans [1].

In April 2009, human infections with a novel influenza A (H1N1) virus were identified, and this virus subsequently caused a worldwide pandemic. The 2009 pandemic influenza A (H1N1) virus is derived from influenza $A$ viruses that have circulated in swine during the past several decades and is antigenically distinct from human influenza A (H1N1) viruses in circulation since 1977. The 2009 pandemic influenza A (H1N) virus contains a combination of gene segments that had not been reported previously in animals or humans. The hemagglutination (HA) gene, which codes for the surface protein most important for immune response, is related most closely to the HA found in contemporary influenza viruses circulating among pigs. This HA gene apparently evolved from the avian-origin 1918 pandemic influenza 
H1N1 virus, which is thought to have entered human and swine populations at about the same time [1]. Immunity to surface antigens, particularly hemagglutinin, reduces the likelihood of infection. Antibody against one influenza virus type or subtype confers limited or no protecttion against another type or subtype of influenza virus. Furthermore, antibody to one antigenic type or subtype of influenza virus might not protect against infection with a new antigenic variant of the same type or subtype. Frequent emergence of antigenic variants through antigenic drift is the virologic basis for seasonal epidemics and is the reason for annually reassessing the need to change one or more of the recommended strains for influenza vaccines. More dramatic changes, or antigenic shifts, occur less frequently. Antigenic shift occurs when a new subtype of influenza A virus appears and can result in the emergence of a novel influenza A virus with the potential to cause a pandemic [1].

The 2009 pandemic influenza A (H1N1) virus is not a new subtype, but because most humans had no pre-existing antibody to key pandemic 2009 influenza A (H1N1) virus hemagglutinin epitopes, widespread transmission was possible [1].

The epidemiology of pandemic (H1N1) 2009. Influenza virus infection indicates that children and young adults have had the highest attack rates [2]. A wide clinical spectrum of disease ranging from non-febrile, mild upper respiratory tract illness, febrile influenza-like illness (ILI) to severe or even fatal complications, including rapidly progressive pneumonia has been described. The most commonly reported symptoms included cough, fever, sore throat, muscle aches, malaise, and headache. Some patients experienced gastrointestinal symptoms (nausea, vomiting, and/or diarrhea). Approximately 10\% $30 \%$ of hospitalized patients in some countries have required admission to intensive care units (ICU). Critically ill patients include those who experienced rapidly progressive lower respiratory tract disease, respiratory failure, and acute respiratory distress syndrome (ARDS) with refractory hypoxemia. Other severe complications have included secondary invasive bacterial infection, septic shock, renal failure, multiple organ dysfunction, myocarditis, encephalitis, and worsening of underlying chronic disease conditions such as asthma, chronic obstructive pulmonary disease (COPD), or congestive cardiac failure. [2-5]. However, data on disease severities in Yemen are scarce. During the pandemic (H1N1) 2009, the Ministry of Health and Population mandated that only patients with moderate to severe cases of influenza-like illness (ILI) be admitted to government (public) and private hospitals for observation and management.

In Yemen, the first laboratory-confirmed case of pandemic influenza was detected on 16, June 2009, while the first fatal case associated with pandemic influenza A
(H1N1) was reported on 17 August 2009.

This study describes the incidence of all deaths cases associated with pandemic influenza A (H1N1) in Yemen. It sets out to highlight the factors associated with poor prognosis to enhance the implementation of prevention and control programs.

\section{METHODOLOGY}

The study is based on retrospective analysis of available data until 14 March 2010, as compiled by the Disease Control and Surveillance team in Yemen. With the onset of the pandemic, an enhanced surveillance system for pandemic influenza A (H1N1) was set up in Yemen, which included direct reporting to the General Directorate of the Diseases Control and Surveillance of each laboratory confirmed case of pandemic influenza A (H1N1) with a fatal outcome. The following data were collected for each case, using a standardized form: demographics (age, sex, residence, and nationality), laboratory findings and time course of illness (date of onset, date of admission, date of death), underlying diseases, and treatment. Trained health care workers extracted data from case notes.

A case of Influenza-like illness (ILI) was defined as a person with sudden onset of fever of $>38^{\circ} \mathrm{C}$ with dry cough or sore throat, in the absence of other known causes.

Fatal cases: were defined as those associated with pandemic influenza A (H1N1), provided that the infection was laboratory-confirmed either before or after death, regardless of the underlying cause of death. With the onset of the pandemic, nasopharyngeal swab specimens collected from each case "under investigation" were sent to either of two reference laboratories: National Public Health Laboratory Center in Sana'a, and NAMRO-3 in Egypt, for quality control. The diagnosis was confirmed by real-time reverse transcription-PCR.

\section{Statistical analysis:}

Frequency tables were used for data presentation and the association between underlying disease and sexes was considered statistically significant at $\mathrm{p}<0.05$ by using chi-squared test. Yemeni population census 2004 was used to calculate incidence [6]. Data was analyzed using SPSS for windows, version 13.0.

\section{Ethical considerations:}

Approval from the academic research committee of the faculty of Medicine of the University of Thamar and Ministry of Public Health and Population in Yemen was obtained prior to data collection.

\section{RESULTS}

Between 16 June 2009 and 14 March 2010, a total of 33 laboratory-confirmed death cases associated with pan- 
demic influenza A (H1N1) were reported to the Diseases Control and Surveillance in the Ministry of Public Health and Population. During this period, a total of 6049 suspected influenza A (H1N1) cases were recorded. With this denominator, the case fatality rate (CFR) was $0.54 \%$. On a general population basis, this corresponds to 1.67 deaths per million according to the Yemeni population census 2004 [6].

During June through August, H1N1 confirmed cases were infrequently detected, including only 30; however, from September through December, over 200 confirmed cases were reported each month, Figure 1.

Of the total 33 cases recorded, 25 were male (76\%) and 8 were female (24\%), male to female Ratio being $3: 1$.

Overall median age of the fatal cases was 30.8 years (range 1 - 55). Eleventh (11) cases (33.3\%) were in the age group 30 - 39 years (Figure 2).

Underlying disease was equally distributed between the sexes, but understandably not among age groups ( $\mathrm{p}=$ 0.001). The most common admission diagnosis was pneumonia. Twenty five (75.8\%) of fatal cases had no documented underlying disease. Chronic cardiovascular disease $(9.1 \%)$ was the most commonly reported term conditions and 2 deaths (6.1\%) were recorded among pregnancy (Table 1).

The median time from onset of symptoms to hospital admission was 2 days (range 1 - 8); while the median time from onset of the influenza likes symptoms to the onset of death was 4 days.

The median length of hospitalization among all fatal cases was 2 days.

Majority of fatal cases (98\%) were prescribed antiviral drugs (osteltamivire). The Median time from the date of antiviral onset to death was 1 day (range 1 - 5 days).

All cases were prescribed antibiotics. The most common antibiotics administered were cefotaxime, ceftriaxone and ciprofloxacine.

\section{DISCUSSIONS AND CONCLUSIONS}

Pandemic influenza A (H1N1) was considerably less lethal than originally expected. In Yemen, the first laboratory-confirmed case of pandemic influenza was detected on 16, June 2009, while the first fatal case associated with pandemic influenza A (H1N1) was reported on 17 August 2009. This study describes the incidence of all deaths cases associated with pandemic influenza A (H1N1) in Yemen. It sets out to highlight the factors associated with poor prognosis to enhance the implementation of prevention and control programs.

The estimation of case fatality rate using the reported cases in a denominator was $0.54 \%$. This finding is consistent with other studies done by Garske T., Vaillant L. and Fajardo-Dolci GE, et al. [7-9]. This result provides an

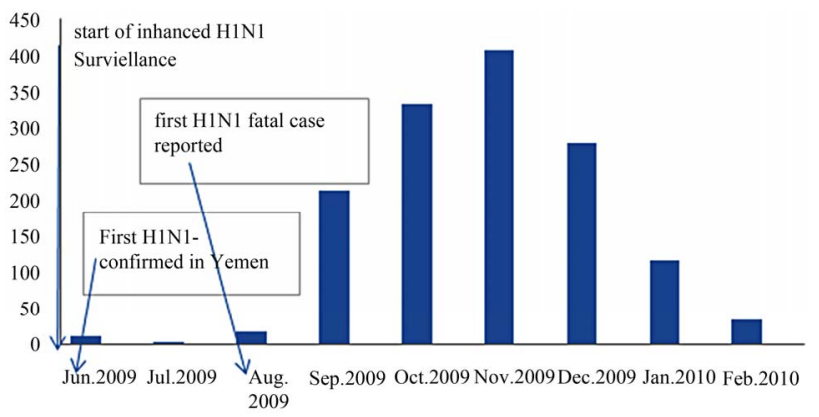

Figure 1. Time (monthly) distribution of pandemic influenza A (H1N1), laboratory confirmed cases.

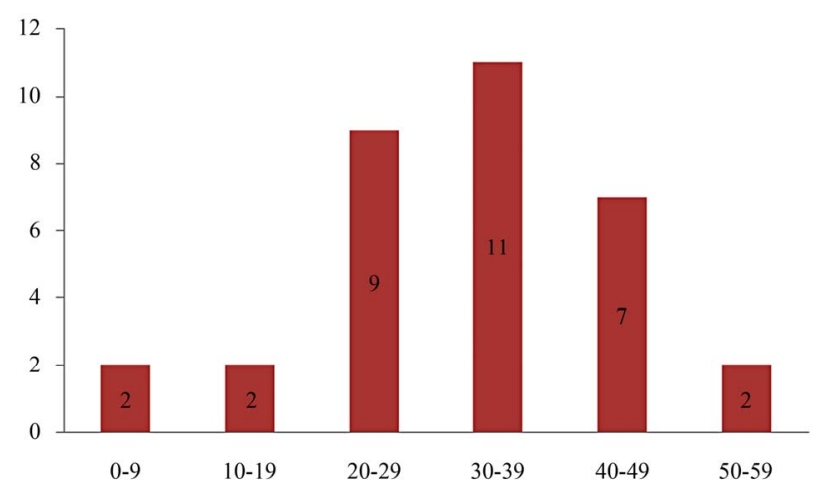

Figure 2. Age distribution of pandemic influenza A (H1N1) fatal cases.

Table1. Distribution of underlying diseases for fatal cases.

\begin{tabular}{ccc}
\hline Underlying disease & Frequency & Percentage (\%) \\
\hline Without any underlying condition & 25 & 75.8 \\
Bronchial asthma & 1 & 3.0 \\
Cardiovascular diseases & 3 & 9.1 \\
Renal failure & 2 & 6.1 \\
Pregnancy & 2 & 6.1 \\
Total & 33 & 100.0 \\
\hline
\end{tabular}

under-estimation of the incidence of all H1N1 cases and therefore an over-estimation of the CFR. The estimated population mortality rate of 1.67 deaths per million is also subject to question due to the number of cases that may have died from H1N1 and have remained undiagnosed. Furthermore, the proportion of undiagnosed cases may vary across different countries.

Regarding the time distribution of fatal cases, it follows an epidemic curve with a peak in the early winter/ November, 2009 in Yemen. This finding is consistent with the median time from onset of symptoms to death (4 days), range (1 - 9).

The male to female ratio was 25:8 = 3:1. These findings disagree with other studies done in China, Europe and Malaysia [10-12]. One possible explanation for this difference could be that women in Yemen are usually 
veiled and this may give them an additional protection against exposure to infection.

The median age of all fatal cases was 30.8 years, and these findings are in agreement with those reported earlier in the USA [13]. The elderly appear to be protected from infection to some extent, but when infected, they are more likely to have a fatal outcome than younger cases.

Our findings on pregnancy and chronic underlying diseases (cardiovascular diseases, bronchial asthma and renal failure) are in agreement with other studies worldwide $[2,13,14]$.

Data regarding the time course of illness are comparable with those reported in other studies [15-17].

\section{Study Limitations:}

One limitation of this study may be under-diagnosis of fatal cases from pandemic influenza H1N1. The difficulty in determining whether the death is attributable to H1N1 infection or to associated factors remains a major limitation too.

\section{Study Recommendations:}

These findings should be taken into consideration when vaccination strategies are employed. Persons at high risk for influenza complications should still be given priority. Regarding the vaccination of the whole population, apart from the fact that a substantial majority of deaths occurred among healthy people, the results of cost benefit analyses should also be taken into account.

\section{AUTHORS' CONTRIBUTIONS}

All authors attest to having contributed substantially to conception and design and acquisition of data and drafting the article and revising it critically for important intellectual content. All authors give approval of the final version to be published.

\section{ACKNOWLEDGEMENTS}

The authors thank all surveillance staff, physicians, nurses and health care workers in the public and private hospitals and intensive care units in Yemen for their kind detections, reporting and observations of all pandemic influenza A (H1N1) cases.

\section{REFERENCES}

[1] Centers for Disease Control and Prevention (2011) Introduction and biology of influenza. http://www.cdc.gov/flu/professionals/acip/background.ht $\underline{\mathrm{m}}$

[2] WHO (2009) Clinical management of human infection with new influenza A (H1N1) virus: Initial guidance. http://www.who.int/csr/disease/swineflu/guidance/surveill ance/WHO_case_definition_swine_flu_2009_04_29.pdf

[3] WHO (2009) Human infection with new influenza A (H1N1) virus: Clinical observations from Mexico and other affected countries. Weekly Epidemiological Record, 21, 185-196. http://www.who.int/wer

[4] Dawood, F.S., Jain, S., Finelli, L., Shaw, M.W., Lindstrom, S., Garten, R.J., Gubareva. L.V., Xu, X., Bridges, C.B. and Uyeki, T.M. (2009) Emergence of novel swineorigin influenza A (H1N1) virus humans. New England Journal of Medicine, 361, 102.

[5] O’Riordan, S., Barton, M., Yau, Y., Read, S.E., Allen, U. and Tran, D. (2010) Risk factors and outcomes among children admitted to hospital with pandemic H1N1 influenza. Canadian Medical Association Journal, 182, 39-44. doi:10.1503/cmaj.091724

[6] (2004)Yemen population census http://www.csoyemen.org/content.php?do=pcat\&cid=1

[7] Garske, T., Legrand, J., Donnelly, C.A., Ward, H., Cauchemez, S., Fraser, C., Ferguson, N.M. and Ghani, A.C. (2009) Assessing the severity of the novel influenza A/ H1N1 pandemic. British Medical Journal, 339, 2840. doi:10.1136/bmj.b2840

[8] Vaillant, L., La Ruche, G., Tarantola, A. and Barboza, P. (2009) Epidemic intelligence team at InVS.Epidemiology of fatal cases associated with pandemic H1N1 influenza 2009. Euro Surveillance, 14, 19309.

[9] Fajardo-Dolci, G.E., Hernández-Torres, F., SantacruzVarela, J., Rodríguez-Suárez, J., Lamy, P., Arboleya-Casanova, H., Gutiérrez-Vega, R., Manuell-Lee, G. and Córdova-Villalobos, J.A. (2009) Epidemiological profile of mortality due to human influenza A (H1N1) in Mexico. Salud Pública de México, 51, 361-371.

[10] Wong, C.M., Yang, L., Chan, K.P., Leung, G.M., Chan, K.H., Guan, Y., Lam, T.H., Hedley, A.J. and Peiris, J.S. (2006) Influenza associated hospitalization in a subtropical city. PLOS Medicine, 3, e121. doi:10.1371/journal.pmed.0030121

[11] European Centre for Disease Prevention and Control (2009) Announced number of new and cumulative confirmed fatal 2009 pandemic influenza A (H1N1) cases in EU and EFTA countries, as of week 17-2010. http://www.ecdc.europa.eu/en/healthtopics/H1N1/Pages/ Reported number of new and cumulative confirmed fatalcases.aspx

[12] Muhammad Ismail, H.I., Tan, K.K., Lee, Y.L., Pau, W.S.C., Razali, K.A.M., Mohamed, T., Adnan, T., Subramaniam P. and J. Hanif (2011) Characteristics of children hospitalized for pandemic (H1N1) 2009, Malaysia. Emerging Infectious Diseases, 17, 708-710. www.cdc.gov/eid

[13] Centers for Disease Control and Prevention (2009) Hospitalized patients with novel influenza A (H1N1) virus infection-California, April-May. Morbidity and Mortality Weekly Report (MMWR), 58, 536-541.

[14] Hewagama, S., Walker, S.P., Stuart, R.L., Gordon, C., Johnson, P.D., Friedman, N.D., O’Reilly, M., Cheng, A.C. and Giles, M.L. (2010) H1N1 influenza A and pregnancy outcomes in Victoria, Australia. Clinical Infectious Diseases, 50, 686-690. doi:10.1086/650460

[15] Lytras, T., Theocharopoulos, G., Tsiodras, S., Mentis, A., Panagiotopoulos, T. and Bonovas, S. (2009) Influenza surveillance report group. Enhanced surveillance of influ- 
enza A (H1N1) in Greece during the containment phase. Euro Surveillance, 14, 19275.

[16] Kumar, A., Zarychanski, R., Pinto, R., Cook, D.J., Marshall, J., Lacroix, J., Stelfox, T., Bagshaw, S., Choong, K., Lamontagne, F., Turgeon, A.F., Lapinsky, S., Ahern, S.P., Smith, O., Siddiqui, F., Jouvet, P., Khwaja, K., McIntyre, L., Menon, K., Hutchison, J., Hornstein, D., Joffe, A., Lauzier, F., Singh, J., Karachi, T., Wiebe, K., Olafson, K., Ramsey, C., Sharma, S., Dodek, P., Meade, M., Hall, R. and Fowler, R.A. (2009) Canadian critical care trials group H1N1 collaborative. Critically ill patients with 2009 influenza A (H1N1) infection in Canada. Journal of the American Medical Association, 302, 1872-1879.

[17] Centers for Disease Control and Prevention (2009) Surveillance for pediatric deaths associated with 2009 pandemic influenza A (H1N1) virus infection-United States, April-August 2009. April-May. Morbidity and Mortality Weekly Report (MMWR), 58, 941-947. 